\title{
Genomic instability and colorectal cancer
}

Colorectal cancer (CRC) includes two primary types of genomic instability: microsatellite instability (MSI) and chromosome instability (CIN). MSI results in a high rate of point mutation, whereas CIN refers to an increased rate of cumulative chromosome disorders (1). Microsatellites are short, tandem-repeat DNA regions that may be found within genes or else, they may constitute non-coding genome regions. MSI occurs in 15\% of CRCs and results from inactivation of the mismatch repair (MMR) system, either from mutation in said genes or from MLH1 gene promoter hypermethylation (MLH1 is a gene within this system) (2). The primary function of the mismatch repair system following replication is to remove base-base mispairs and insertion/deletion loops brought about by DNA-polymerase slips during DNA synthesis (3).

Presumably, a defective repair of mispairs encourages malignant transformation by allowing rapidly cumulative mutations to inactivate genes that usually play key roles within cells. Mispair repair defective genes seemingly promote mutations in other genes as well, as they may not produce nucleotide-mispair correcting proteins during DNA replication. However, genes including microsatellites in their own coding sequence are also involved (4).

A hereditary form of colorectal cancer, hereditary non-polypoid colorectal cancer (HNPCC) or Lynch syndrome, represents 3 to 5\% of all CRCs, and is the most common condition within hereditary CRC syndrome. In this syndrome the source of microsatellite instability includes mutations in MMR genes (5). Predisposition to cancer, seen not only in HNPCC but also in other cancerigenic syndromes resulting from germinal mutations in genes regulating DNA fidelity [e.g. Li-Fraumeni syndrome (TP53, CHK2), Nijmegen syndrome (NBS1), Bloom syndrome (BLM), and ataxia telangiectasia (ATR/ATM)], demonstrates that inactivation mechanisms regulating genome stability play a primary role in carcinogenesis (2). MMR system-deficient cells exhibit a mutating phenotype with highly increased spontaneous mutation rates, which may be 100 to 1000 times greater when compared to normal cells $(3,6)$.

Virtually all CRCs exhibit either MSI or CIN, which suggests that genomic instability is not only common but also crucial in the genesis of CRC. CIN is the most common type of genomic instability in CRC, and occurs in around $85 \%$ of colonic tumors. However, despite the high frequency of CIN in CRC, and the fact that aneuploidy has been long considered a hallmark of cancer, our understanding of this chromosomal disturbance state is still rudimentary (2). Aneuploidy is characterized by changes in the structure and number of chromosomes (7). Among mechanisms that may mediate CIN, genes involved in the detection of interactions between the spindle and kinetochore during mitosis are best candidates to playing a role in chromosomal instability in human cancers (2). The list of eligible genes able to cause CIN exceeds 100 , and includes genes used in kinetochore structure and function; in centrosome and 
microtubules formation and behaviour; in chromosome condensation; in sister chromatides cohesion; and in cell cycle checkpoint regulation (8). In addition to the inactivation of proteins regulating mitotic spindle checkpoints, the inactivation of proteins regulating DNA damage checkpoints, chromosome metabolism, and centrosome functions have been seen to influence chromosome instability in vitro (2). CIN increases the rate at which gross chromosomal changes occur during cell division (9). According to Greenwood (10), cell division is a dangerous issue, since each kinetochore must bind the mitotic spindle so that both chromatides correctly separate towards the opposing cell poles. The integrity of this process is carefully monitored by the spindle assembly checkpoint, and it has been suggested that a defective checkpoint may lead to aneuploidy, as is commonly the case in human cancer.

The article by Cabrera et al. (11) included in this issue of the Journal refers to a scarcely known function of the adenomatous polyposis coli (APC) tumor suppressing gene: its role in genomic instability. This is a gene with multiple functions, including: a) beta-catenin signalling regulation; b) cell adhesion regulation via beta-catenin and E-cadherin; c) cell migration regulation through interaction with microtubules; d) cell cycle blockade maybe through direct cell component inhibition; and e) coordinated cell adhesion and motility regulation (12). However, the first function mentioned is the most acknowledged and common. When the APC gene has mutations resulting in the protein's inability to regulate beta-catenin signals, discontrolled cell division ensues within colonic crypts, which triggers a number of steps that ultimately converge in the development of an adenoma, and then of a malignancy. The paper by Cabrera et al. discusses not only the role of APC gene in various cell processes, including proliferation, differentiation, apoptosis, adhesion, migration, and chromosome segregation, but also provides insight into the mutational analysis of this gene, as well as the implications of mutations in the development of the adenoma-carcinoma sequence. Regarding its role in chromosome instability, the authors state that APC mutant cells have many microtubules unable to bind the kinetochore, and are therefore responsible for the chromosome instability phenotype seen in these cells. Similarly, following a discussion of results from experiments using transgenic mice able to reproduce CIN, they prove how the selective benefit of colonic tumor cells manifests itself when they lose beta-catenin-dependent function, and hence the resulting chromosome instability is a consequence of this loss, not the tumor-triggering process.

Furthermore, these authors report that a number of human colon cancer cell lines that are well characterized with APC mutations have no chromosome instability following thousands of cell divisions in vitro, and they conclude that APC inactivation alone is unlikely to result in a CIN phenotype in human colorectal cancer, whereas other genes are likely involved in such process.

D. Cruz-Bustillo Clarens

National Institute of Gastroenterology. Havana, Cuba

\section{REFERENCES}

1. Michor F, Iwasa Y, Lengauer C, Nowak MA. Dynamics of colorectal cancer. Seminars in Cancer Biology 2005 (in press).

2. Grady WM. Genomic instability and colon cancer. Cancer and Metastasis Reviews 2004; $23: 11-27$.

3. Peltomaki P. DNA mismatch repair and cancer. Mut Res 2001; 488: 77-85. 
4. De la Chapelle A. Microsatellite instability. N England J Med 2003; 349: 209-10.

5. Gra Menéndez S, Cruz-Bustillo Clarens D. Algunos aspectos genéticos, moleculares y clínicos del cáncer colorrectal hereditario no polipoideo. Rev Cubana Invest Biomed 2004; 22: 43-50.

6. Schofield MJ, Hsieh P. DNA mismatch repair: Molecular mechanisms and biological function. Annu Rev Microbiol 2003; 57: 579-608.

7. Rajagopalan H, Nowak MA, Vogelstein B, Lengauer C. The significance of unstable chromosomes in colorectal cancer. Nat Rev Cancer 2003; 3: 695-701.

8. Jallepalli PV, Lengauer C. Chromosome segregation and cancer: Cutting through the mystery. Nat Rev Cancer 2001; 1: 109-17.

9. Komarova NL, Lengauer C, Vogelstein B, Nowak MA. Dynamics of genetic instability in sporadic and familial colorectal cancer. Cancer Biol Therapy 2003; 6: 685-92.

10. Greenwood E. Genomic instability: Dangerous division. Nat Rev 2003; 3: 148.

11. Cabrera CM, López-Nevot MA. APC e inestabilidad cromosómica en el cáncer de colon. Rev Esp Enferm Dig 2005; 97 (10): 738-43.

12. Cruz-Bustillo Clarens D. Genética molecular del cáncer colorrectal. Rev Esp Enferm Dig 2004; 96: 48-59. 